\title{
Kritische Bemerkungen
}

zum Aufsatz , Der Positioner - Ein Ersatz für das Einschleifen?"،

von G. Schrems-Adam und H. Th. Schrems

Fortschritte der Kieferorthopädie, Bd. 37, 1976, Heft 4, S. 473-478

\section{Von Anton Baldauf. München}

In dem Artikel „,Der Positioner - ein Ersatz für das Einschleifen?" von SchremsAdam und Schrems in Fortschritte der Kieferorthopädie 37(1976), 473 scheint mir ein wichtiger Punkt nicht beachtet worden zu sein. $U$. a. will man mit einem gnathologischen Positioner erreichen, daß der in terminaler Scharnier-Relation befindliche Unterkiefer bei der Schließbewegung in maximale Interkuspidation schließt. Dadurch aber, daß die Autoren alle Zähne aus den Modellen heraussägen, geht die terminale Scharnier-Relation im Artikulator verloren - die Artikulatormontage verliert ganz beträchtlich an Wert. Um die terminale ScharnierRelation im Artikulator zu bewahren, ist es notwendig, im Ober- und Unterkiefermodell Schlüsselzähne stehen zu lassen. Nur dann wird der Positioner den Unterkiefer des Patienten nicht aus der terminalen Scharnier-Relation herauszwingen.

Mit dem Vorgehen der Autoren wird m. E, nicht gezielt auf das gnathologische Behandlungsziel hingearbeitet.

Anschr. d. Verf.: Dr. Arton Baldauf, Faberstrafle 11, D-8000 Munchen 70 :

\section{Stellungnahme zu den obigen Bemerkungen}

\section{Von H. Th. Schrems und G. Schrems-Adam, Regensburg}

Die von Hern Kollegen Baldauf geäußerte Vermutung, daß durch das Heraussăgen der Zâhne aus den Modellen die 'terminale Scharnier-Relation' vertoren gehe und die Artikulatormontage damit ganz betrachtlich an Wert verlore, ist nicht zutreffend.

Die terminale Scharnierachsenposition ist definiert als diejenige Lage, bei der die Scharnierachse sich in der retralen und kranialen Lage befindet, die Kondylen in nicht seitenverschobener Position. Die scheinbaren Durchtrittsstellen der Achse durch die Haut dienen dabei als Referenzpunkte. (Gemeinsame Nomenklaturvorschläge des Arbeitskreises Funktionelle Gebißanalyse der Deutschen Gesellschaft für Zahn-, Mund- und Kieferheilkunde und der Nomenklaturkommission der Deutschen Gesellschaft für Zahnärztliche Prothetik und Werkstoffikunde, vgl. Dtsch. zahnärztl. Z. 31 (1976), V)

Wir haben in unserem Aufsatz ausdrucklich zweimal ausgeführt, dab wit die Modelle im Artikulator in der terminalen Scharnierachsenposition montieren, weil in dieser Position Ober- und Unterkiefer unabhängig von der vorliegenden Okklusion reproduzierbar zugeordnet werden konnen. Die terminale Scharnier- 